\title{
Transmission of microwave radiation through a sub-wavelength slit with internal structure
}

\author{
Matthew J. Lockyear, Alastair P. Hibbins and J. Roy Sambles \\ Electromagnetic Materials Group, School of Physics, University of Exeter, \\ Exeter EX4 4QL, UK
}

\begin{abstract}
Fabry-Perot cavities are perhaps the best known of the optical transmission resonators, with cavity field enhancement accomplished by two parallel and partially reflecting planes. Recently it has been shown that arrays of narrow slits cut into a metal substrate are similarly able to exhibit resonant transmission modes. Here, the transmission of normally incident plane wave microwaves through a single stepped sub-wavelength slit in a thick metal plate is explored. The presence of the step substantially increases the radiation wavelength, which may be resonantly transmitted to well beyond twice the plate thickness. Insight into the resonant behaviour of the stepped slit is provided through the analysis of the field solutions produced by a finite element model. This model also predicts resonant transmission which is in excellent agreement with the experimental results.
\end{abstract}

Keywords: Fabry-Perot, sub-wavelength slit, waveguide mediated transmission, etalon

\section{INTRODUCTION}

In recent years there has been much interest in the 'enhanced' transmission phenomena of sub-wavelength apertures in metals, structured or otherwise [1-15]. Unlike sub-wavelength holes, which rely on either diffraction or surface wave excitation to enhance the evanescent tunnelling process through the holes, slits support a propagating transverse electromagnetic (TEM) waveguide mode without cut off [16]. This mode gives strong transmission through such structures at well defined wavelengths [8].

For a single sub-wavelength slit in a thick metal substrate, weak coupling to the TEM occurs at the slit aperture due to diffraction of the incident field. Radiation, once within the slit, does not couple efficiently back out into free space due to a mode-shape mismatch, resulting in the open ends of the slit acting as highly reflecting mirrors which can support a series of standing waves. It is these Fabry-Perot (FP) like modes that provide strong resonant enhancement at selective wavelengths to an otherwise weak transmission. In the limit of infinitesimal slit width and perfect conductors, peaks in the transmission will be found according to;

$$
\lambda_{N}=\frac{2 n l}{N}
$$

where $\lambda_{N}$ is the wavelength of the $N^{\text {th }}$ order mode, $n$ is the refractive index within the slit and $l$ is the thickness of the metal plates (separation between the two partially reflecting planes). (Real structures exhibit a generally small reduction in this predicted frequency $[9,17]$ through two effects, one an end effect due to the finite width of the slit, the other due to wavefront curvature within the slit through the finite conductance of the metal.) In the ideal case through quantisation of the mode along the slit length, there is an upper limit of approximately twice the sample thickness (times the refractive index of the 
material filling the slit) for the longest wavelength transmission resonance. Here we show that the addition of a suitable step structure within the slit results in the resonant wavelength of the fundamental mode being dramatically increased.

\section{EXPERIMENTAL}

The three experimental samples studied here are based upon an air-filled slit formed between two aluminium plates of dimensions $400 \mathrm{~mm}(x) \times 300 \mathrm{~mm}(y) \times 10 \mathrm{~mm}(z)$. When arranged with $y z$ faces aligned and separated by incompressible polyester spacers in the $z$ direction, a slit of length $l=10 \mathrm{~mm}$, breadth $b=300 \mathrm{~mm}$, and slit width $w$ is formed in an otherwise opaque metal screen (Figure 1a). The reference sample (A) consists of a sub-wavelength slit with no additional structuring. Sample (B) is identical to sample (A) with the exception of a $3 \mathrm{~mm}$ wide, $0.36 \mathrm{~mm}$ deep step milled into the $y z$ face of one of the substrates that extends throughout the entire breadth of the sample $(300 \mathrm{~mm})$. Sample (C) has a notch milled into both surfaces (Figure 1c).
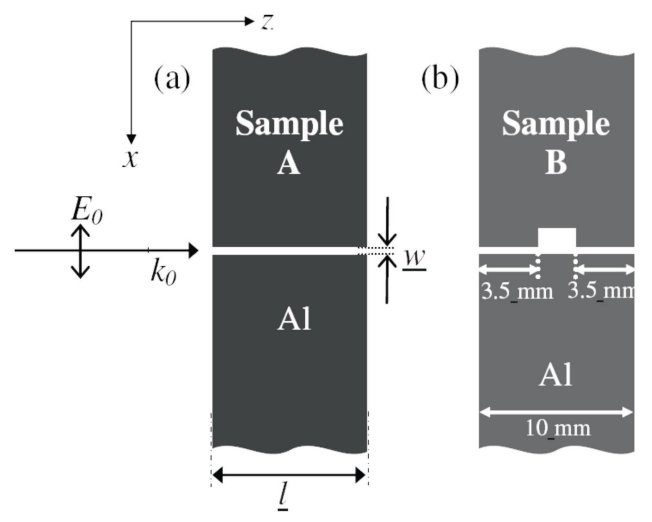

(c)

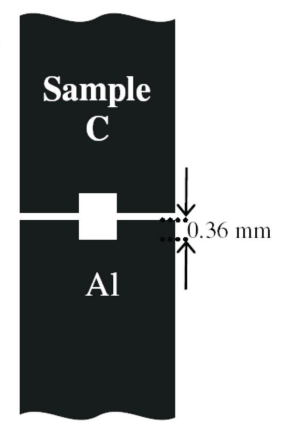

FIG. 1. The three experimental samples under study. Sample A consists of a subwavelength slit formed by the arrangement of two thick aluminium plates, separated at the ends by Mylar spacers. Samples B and C are variations of sample A, containing a rectangular step $3.5 \mathrm{~mm}$ deep and $3 \mathrm{~mm}$ wide, positioned centrally between the exit and entrance apertures of the slit.

The experimental arrangement utilises a spherical mirror (of focal length 2 meters) to collimate microwave radiation from a horn antenna and direct it onto the sample at normal incidence. Incident radiation is linearly polarised with its $E$-vector orientated in the $x$ direction (normal to the slit). The beam width is less than the width $\mathrm{b}$ of the samples so they are effectively infinite in the y-direction. The transmitted radiation is collected by a second identical mirror and directed to a matched detector horn. In this way transmission is recorded as a function of frequency for different slit widths for all three samples.

\section{RESULTS AND DISCUSSIONS}

Figure 2 inset shows the relative transmission (circles) of sample (A) as a function of frequency and for a range of slit widths between $75 \mu \mathrm{m}$ and $600 \mu \mathrm{m}$ in $25 \mu \mathrm{m}$ increments. Note the strong resonant features which compare in position and form very well with the predictions from a finite element method (FEM) modelling code [18] (solid lines). The calculated fields at the resonant frequencies (Figure 5) show these transmission maxima all to be the fundamental FP mode. We note the narrowing of the resonance and the reduced transmission with decreasing slit width. However of more interest in this study is the 
frequency of the fundamental resonance (Figure 2). It is clear that for this slit, without structure, the resonant frequency decreases monotonically with increasing slit width due to an aperture effect [9].

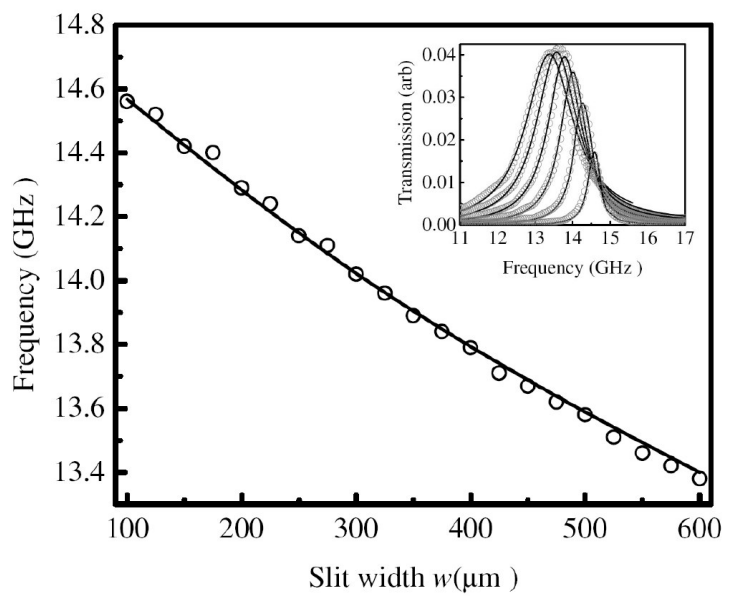

FIG. 2 . Resonant frequency as a function of slit width $w$ for the fundamental resonance of sample A (circles). Also shown are the predictions of a finite element method model (solid line). Inset: The modelled and experimental transmission spectra for the fundamental resonance of sample A, recorded for six values of slit width between $100 \mu \mathrm{m}$ (highest frequency resonance) and $600 \mu \mathrm{m}$ (lowest frequency resonance) in $100 \mu \mathrm{m}$ increments.

The resonant frequency of the fundamental mode of sample (B) (circles) as a function of slit width is displayed in Figure 3 together with the modelled response. On comparison of Figure 2 with Figure 3 it is clear that the addition of the step to one of the metallic substrates dramatically changes the resonant behaviour observed. Rather than reducing monotonically with increasing slit width, the fundamental resonance supported by sample (B) increases in frequency.

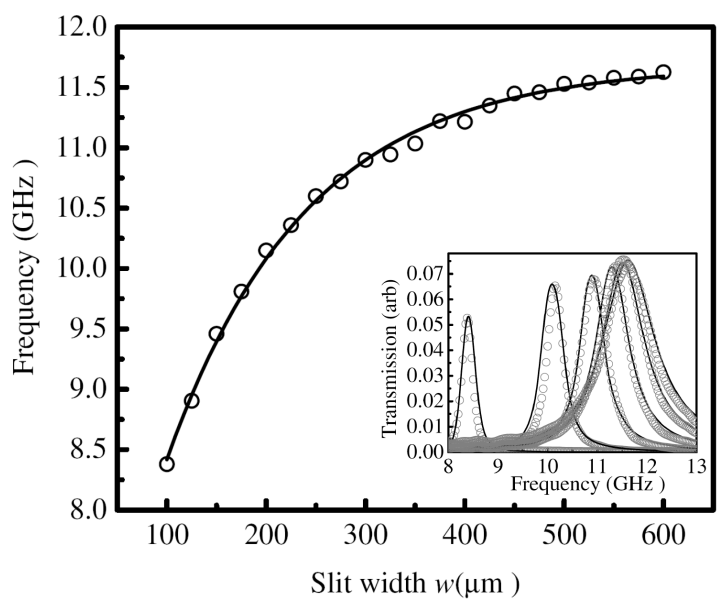

FIG. 3. Resonant frequency as a function of slit width $w$ for the fundamental resonance of sample B (circles) together with modelled results (solid line). Inset: The modelled and experimental transmission spectra for the fundamental resonance of sample B, recorded for six values of slit width between 100 and $600 \mu \mathrm{m}$ in $100 \mu \mathrm{m}$ increments.

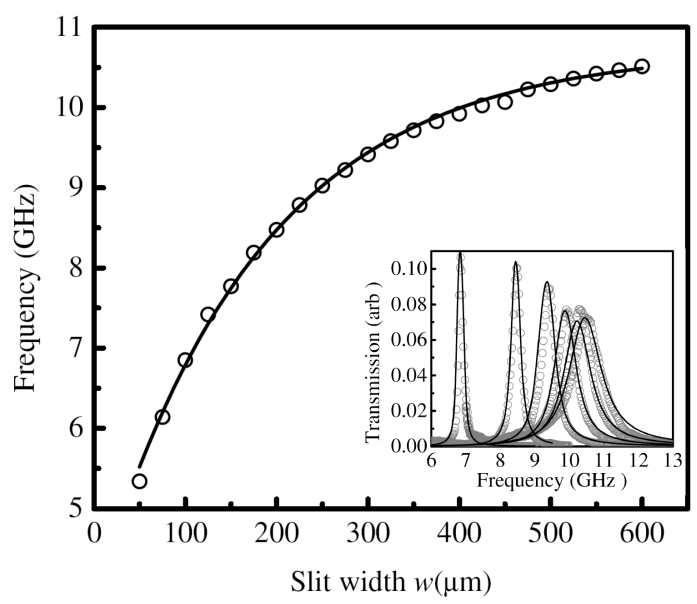

FIG. 4. Resonant frequency as a function of slit width $w$ for the fundamental resonance of sample $\mathrm{C}$ (circles) together with modelled results (solid line). Inset: The modelled and experimental transmission spectra for the fundamental resonance of sample $\mathrm{C}$, recorded for six values of slit width between 100 and $600 \mu \mathrm{m}$ in $100 \mu \mathrm{m}$ increments. 
Indeed, the resonant frequency of the fundamental mode supported by sample (B) is always lower than that of sample (A), for any given slit width. For example, the fundamental resonance of sample (A) occurs at $14.6 \mathrm{GHz}$ when $w=100 \mu \mathrm{m}$, whereas the same slit width for sample (B) gives the resonance at $8.4 \mathrm{GHz}$. This striking reduction is enhanced further with sample C which has an identical step in the other metallic substrate (Figure 4). Remarkably, at a slit width of $100 \mu \mathrm{m}$, the resonant frequency of the fundamental FP like mode has been reduced by 53\% compared to the un-structured case. In order to understand the effect of the additional structure within the slit, it is useful to investigate the electromagnetic response of the structure on resonance using the FEM model.

\section{FINITE ELEMENT METHOD MODELLING}

Figures 5 (a) and (b) show the modelled $E_{x}$ and $H_{y}$ field respectively, calculated on a line passing midway between and parallel to the two metallic substrates of sample (A), and extending $3 \mathrm{~mm}$ beyond each end of the slit. The field strengths are represented in terms of an enhancement over the injected field strength, and calculated at a phase corresponding to maximum enhancement. Grey dotted lines signify the position of the entrance and exit apertures of the sub-wavelength slit.
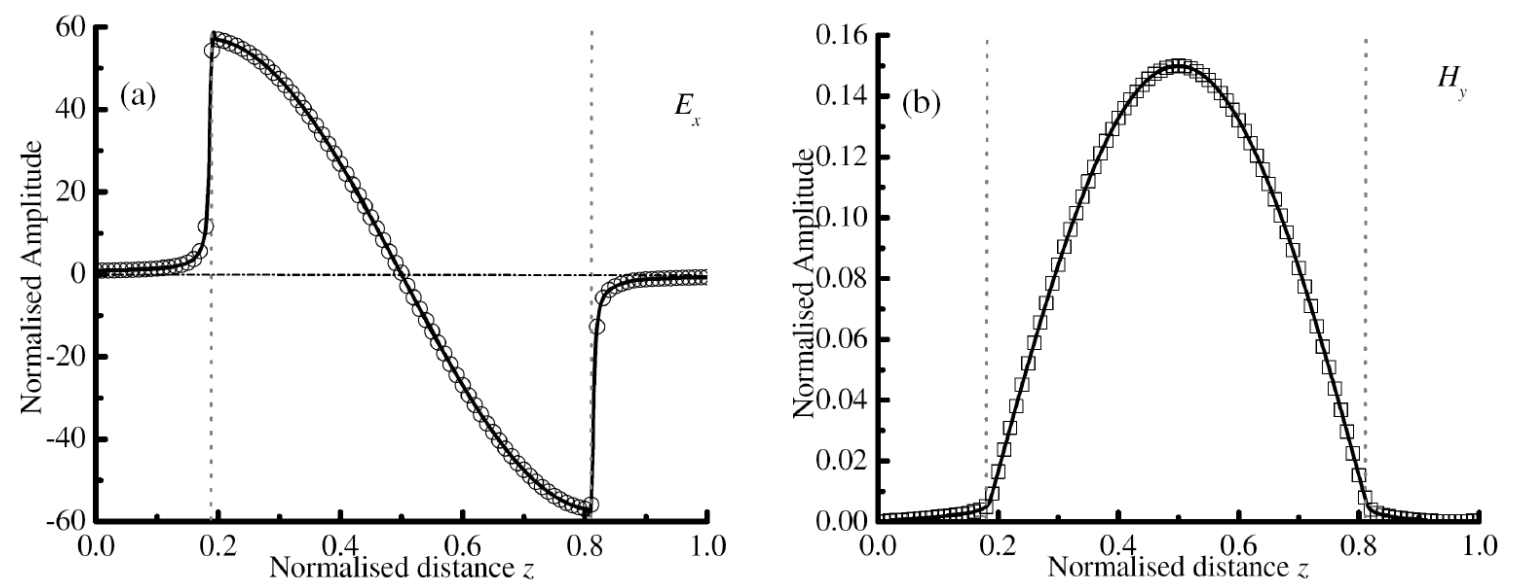

FIG. 5. (a) and (b) show the modelled electric and magnetic field enhancement respectively within the slit region of sample A. Field enhancements are calculated at a phase corresponding to maximum enhancement over a line passing midway between the metallic planes constituting the slit and extends $3 \mathrm{~mm}$ past the slit apertures. The vertical dotted lines are representative of the position of the apertures of the slit.

Firstly we note that the $\mathrm{E}$ field strength has a maximum at the apertures of the slit, whilst the $\mathrm{H}$ field strength has a maximum in the central region of the slit. This is as one would expect given 'magnetic- wall' boundary conditions at each end of the slit $[19,20]$, and the standing wave nature of the resonance supported (i.e. the maxima in $\mathrm{E}$ and $\mathrm{H}$ are separated by $\sim \pi / 4$ radians of phase both spatially and temporally). Further, it is in the region of the slit apertures that the greatest gradient in the E field strength occurs. In this region the incident plane wave is matched to the mode within the slit, via the strong field gradient associated with diffraction at the slit aperture. There are only two non-zero components of the incident field along the mid plane of the slit $E_{x}$ and $H_{y}$. However this is not the case at the slit apertures. In this region, the strong diffraction of the 
incident field into the slit gives rise to a non-zero $E_{z}$ component, and also a derivative of $E_{z}$ with respect to $y$. In this region Maxwell's equation $\underline{\nabla} \wedge \underline{E}=-\mu \partial H / \partial t$, which may be written as

$$
\left|\frac{\partial E_{z}}{\partial y}-\frac{\partial E_{y}}{\partial z}\right|=-\frac{2 \pi c \mu}{\lambda}\left|H_{x}\right|
$$

is solved with a much faster gradient of fields than is otherwise allowed in free space. This is because both $\frac{\partial E_{z}}{\partial y}$ and $\frac{\partial E_{y}}{\partial z}$ are very large but nearly cancel, varying rapidly over a distance of the order of the width of the slit (which is much smaller than the incident wavelength). The $\pi / 2$ phase change required to satisfy the matching of the cavity mode and the plane wave propagating in free space is then compressed into a distance of the order of the slit width. (A comprehensive investigation of the fields and boundary conditions associated with an array of such un-structured metallic slits by the current authors may be found in reference [19] together with a comparison to the more conventional etalon.) However it is a similar type of "fieldexpansion' at the steps within the slit which gives rise to the effective lengthening of the stepped slits.
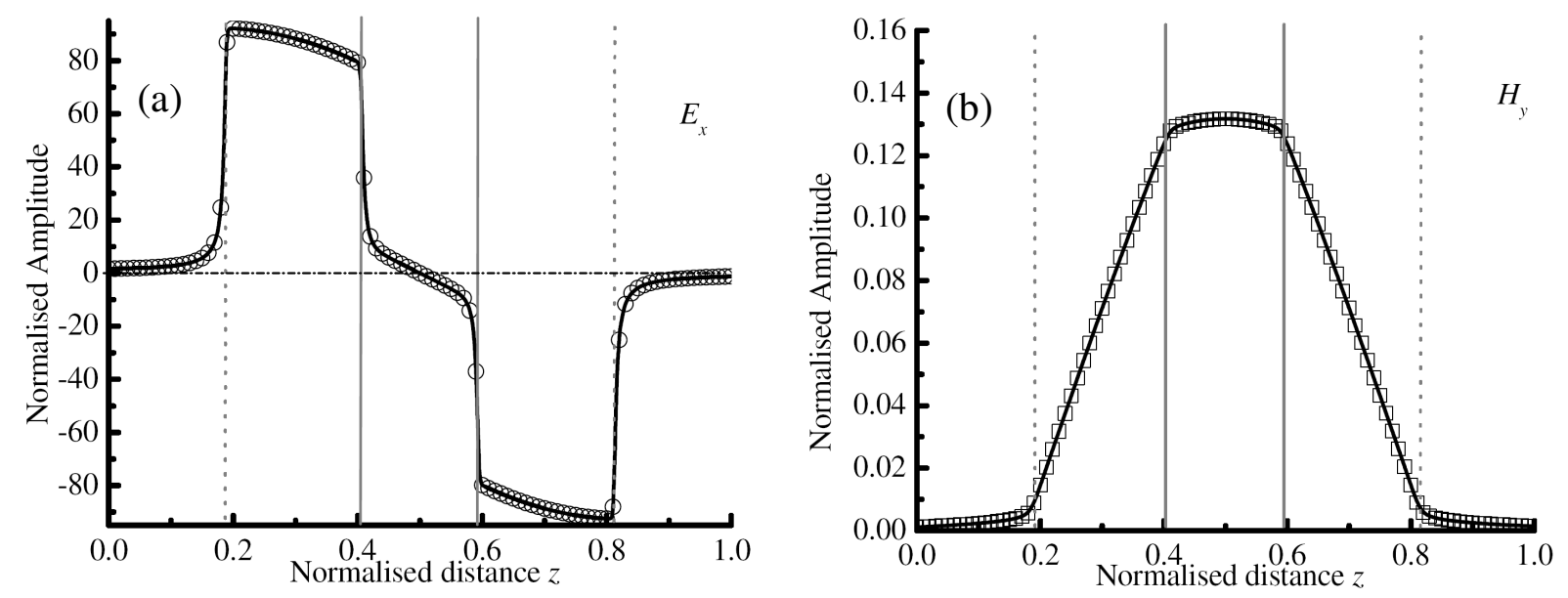

FIG. 6. (a) and (b) show the modelled electric and magnetic field enhancement respectively within the slit region of sample $\mathrm{C}$. The vertical dotted lines are representative of the position of the apertures of the slit, whereas the solid vertical lines mark the beginning and end of the stepped cavity.

Figure 6 (a) and (b) shows the predicted $\mathrm{E}$ and $\mathrm{H}$ field enhancement respectively that are associated with sample $\mathrm{C}$ (steps in both substrates positioned centrally within the slit) at the resonant frequency of the fundamental mode $(6.85 \mathrm{GHz})$. Once again field strengths are calculated at a phase corresponding to maximum field enhancement with the grey dotted lines indicating the position of the slit exit and entrance apertures. In addition, the solid grey lines of Figure 6 mark the position of the entrance and exit apertures of the central cavity formed by the two notches. On comparison of Figures 5 and 6 we note several striking differences. Firstly, the E and $\mathrm{H}$ fields are now clearly separated spatially, with the E field being strongly confined within the two narrow sections of the slit (the highly capacitive regions) and a region of nearly constant high $\mathrm{H}$ field existing within the stepped region. Importantly, the addition of the steps results in two additional regions over which steep field gradients are obtained. These field gradients are again associated with the diffraction and expansion of the field at either end of the stepped section of the cavity. As the field expands into the wider region of the cavity, they match up with a longitudinal oscillation of surface current density existing on each metallic wall [19]. At any instant the current flow on each 
of the two metallic surfaces must be in opposite directions, forming an inductive current loop within the stepped region thereby increasing the effective inductance of the system. This increase in inductance results in a reduction of the resonant frequency of the mode, since from lumped circuit theory, the notched slit may be thought of as a capacitive part $\mathrm{C}$ (the narrow entrance and exit channels of the slit) and an inductive part $L$ (the stepped cavity) with a resonant frequency to first order given by $1 / \sqrt{L C}$.

\section{CONCLUSIONS}

To summarise, it is shown that with the addition of a suitable step, the fundamental resonant wavelength of a sub-wavelength slit may be increased by approximately 50\%. This shows that suitable internal structuring of sub-wavelength slits and possibly other similar resonant cavities is a route to reducing the dimensions of EM filters or increasing the operational bandwidth of truncated guides.

The authors acknowledge the support of the EPSRC.

\section{REFERENCES}

[1] Ebbesen, T. W., Lezec, H. J., Ghaemi, H. F., Thio, T., and Wolff, P. A., "Extraordinary optical transmission through sub-wavelength hole arrays", Nature 391, 667-69 (1998).

[2] Ghaemi, H. F., Thio, T., Grupp, D. E., Ebessen, T. W., and Lezec, H. J., "Surface plasmons enhance optical transmission through subwavelength holes", Phys. Rev. B 58, 6779 (1998).

[3] Lopez-Rios, T., Mendoza, D., Garcia-Vidal, F. J., Sanchez-Dehesa, J., and Pannetier, B., "Surface shape resonances in lamellar gratings", Phys. Rev. Lett. 81, 665 (1998).

[4] Schroter U. and Heitmann, D., "Surface-plasmon-enhanced transmission through metallic gratings", Phys. Rev. B. 58, 15419 (1998).

[5] Porto, J. A., Garcia-Vidal, F. J., and Pendry, J. B., "Transmission resonances on metallic gratings with very narrow slits", Phys. Rev. Lett. 83, 2845 (1999)

[6] Lalanne, P., Hugonin, J., Astilean, S., Palamaru, M., and Moller, K., "One-mode model and Airy-like formulae for one-dimensional metallic gratings", J. Opt. A, Pure Appl. Opt. 2, 48 (2000).

[7] Popov, E., Neviere, M., Enoch, S., and Reinisch, R., "Theory of light transmission through subwavelength periodic hole arrays", Phys. Rev. B. 62, 16100 (2000).

[8] Went, H. E., Hibbins, A. P., Sambles, J. R., Lawrence, C. R., and Crick, A. P., "Selective transmission through very deep zero-order metallic gratings at microwave frequencies", Appl. Phys. Lett. 77, 2789 (2000).

[9] Takakura, Y., "Optical resonance in a narrow slit in a thick metallic screen”, Phys. Rev. Lett. 86, 5601 (2001). 
[10] Yang, F. and Sambles, J. R., "Resonant transmission of microwaves through a narrow metallic slit", Phys. Rev. Lett. 89, 063901 (2002).

[11] Hibbins, A. P., Sambles, J. R., and Lawrence, C. R., "Gratingless enhanced microwave transmission through a subwavelength aperture in a thick metal plate", Appl. Phys. Lett. 81, 4661 (2002).

[12] Stavrinou, P. and Solymar, L., "The propagation of electromagnetic power through subwavelength slits in a metallic grating", Opt. Commun. 206, 217 (2002).

[13] Lezec, H. J., Degiron, A., Devaux, E., Linke, R. A., Martin-Moreno, L., Garcia-Vidal, F. J., and Ebbesen, T. W., "Beaming light from a subwavelength aperture", Science 297, 820 (2002).

[14] Martin-Moreno, L., Garcia-Vidal, F. J., Lezec, H. J., Degiron, A., and Ebbesen, T. W., "Theory of highly directional emission from a single subwavelength aperture surrounded by surface corrugations", Phys. Rev. Lett. 90, 16, 167401 (2003).

[15] Lockyear, M. J., Hibbins, A. P. and Sambles, J. R., "Microwave transmission through a single subwavelength annular aperture in a metal plate", Phys. Rev. Lett. 94, 19, 193902 (2005).

[16] Collin, R. E., [Field Theory of Guided Waves], 2nd ed. IEEE, New York (1991).

[17] Suckling, J. R., Hibbins, A. P., Lockyear, M. J., Preist, T. W., Sambles, J. R. and Lawrence, C. R., "Finite conductance governs the resonance transmission of thin metal slits at microwave frequencies", Phys. Rev. Lett. 92, 147401 (2004).

[18] Ansoft's High Frequency Structure Simulator (www.ansoft.com).

[19] Hibbins, A. P., Lockyear, M. J. and Sambles, J. R., "The resonant electromagnetic fields of an array of metallic slits acting as Fabry-Perot cavities", J. Appl. Phys. 99, 124903 (2006).

[20] Sievenpiper D., Zhang L. J., Broas, R. F. J., Alesopolous, N. G., and Yablonovitch, E. "High impedance electromagnetic surfaces with a forbidden frequency band", IEEE Trans. Microwave Theory Tech. 47, 2059 (1999). 